\title{
OPEN Hemangiosarcoma cells induce M2 polarization and PD-L1 expression in macrophages
}

\author{
Kevin Christian M. Gulay ${ }^{1}$, Keisuke Aoshima ${ }^{1 凶}$, Naoya Maekawa² ${ }^{2}$ Tamami Suzuki $^{1}$, \\ Satoru Konnai ${ }^{2,3}$, Atsushi Kobayashi ${ }^{1} \&$ Takashi Kimura $^{1}$
}

Hemangiosarcoma (HSA) is a malignant tumor derived from endothelial cells. Tumor-associated macrophages are one of the major components of tumor microenvironment and crucial for cancer development. The presence and function of macrophages in HSA have not been studied because there is no syngeneic model for HSA. In this study, we evaluated two mouse HSA cell lines and one immortalized mouse endothelial cell line for their usefulness as syngeneic models for canine HSA. Our results showed that the ISOS-1 cell line developed tumors with similar morphology to canine HSA. ISOS-1 cells highly expressed KDM2B and had similar KDM2B target expression patterns with canine HSA. Moreover, we determined that in both ISOS-1 and canine HSA tumors, macrophages were present as a major constituent of the tumor microenvironment. These macrophages were positive for CD204, an M2 macrophage marker, and express PD-L1, an immune checkpoint molecule. Canine HSA with macrophages expressing PD-L1 had a smaller number of T-cells in tumor tissues than tumors with PD-L1 negative macrophages. ISOS-1-conditioned medium could induce M2 polarization and PD-L1 expression in RAW264.7 mouse macrophage cell line and mouse peritoneal macrophages. These results show that ISOS-1 can be used as a syngenic model for canine HSA and suggest that macrophages play an important role in immune evasion in HSA. Using the syngeneic mouse model for canine HSA, we can further study the role of immune cells in the pathology of HSA.

Hemangiosarcoma (HSA) is a rapidly growing and highly invasive endothelial cancer ${ }^{1}$. It is the most common splenic neoplasm in dogs where it usually develops at 6 to 17 years of age ${ }^{2}$. Middle to large breed dogs are most commonly afflicted with $\mathrm{HSA}^{2}$. HSA also occurs, albeit infrequently, in cats, horses, mice, and humans ${ }^{3-6}$. An effective treatment for HSA is difficult to develop since little is known about its molecular pathology. Recently, we found that canine HSA highly expressed three histone demethylases (KDM1A, KDM2A and KDM2B) out of which KDM2B was necessary for HSA cell survival by positively regulating the DNA damage response system in tumor cells ${ }^{7}$. KDM2B silencing not only dysregulated DNA damage responses but also induced expressions of the genes related to inflammatory responses ${ }^{7}$. Inhibiting KDM2B could be an option to induce host immune responses against HSA tumor cells; however, we were unable to investigate the function of KDM2B on immune responses in canine HSA because the immunodeficient mouse model was not suitable to study the immune responses ${ }^{8}$. Syngeneic mouse models, otherwise known as allograft mouse tumor models, are composed of tumor tissues derived from the same genetic background as the mouse strain. They can develop tumors in a fully immunocompetent environment, which can facilitate the examination of the immune-tumor cell interactions. A syngeneic model for HSA, however, is not currently available.

At present, there are few established mouse HSA cell lines such as ISOS-1 and UV $ᄋ$ 2. ISOS-1 was established from a tumor formed by the xenotransplantation of a human angiosarcoma cell line, while UV 2 cell line was developed from an ultraviolet light-induced angioendothelioma-like tumor ${ }^{9,10}$. Their usefulness as syngeneic models for canine HSA or human angiosarcoma, however, has not yet been evaluated.

Macrophages have two states, M1 and M2. M1 macrophages which have anti-tumor effects can kill tumor cells and present tumor antigens to $\mathrm{CD} 4^{+} \mathrm{T}$ cells ${ }^{11}$. M2 macrophages have pro-tumor effects and promote tumor growth and immune evasion by inducing angiogenesis and producing anti-inflammatory cytokines such as IL-3,

\footnotetext{
${ }^{1}$ Laboratory of Comparative Pathology, Department of Clinical Sciences, Faculty of Veterinary Medicine, Hokkaido University, Kita 18 Nishi 9, Kita-ku, Sapporo, Hokkaido 060-0818, Japan. ${ }^{2}$ Department of Advanced Pharmaceutics, Faculty of Veterinary Medicine, Hokkaido University, Sapporo, Hokkaido 060-0818, Japan. ${ }^{3}$ Laboratory of Infectious Diseases, Department of Disease Control, Faculty of Veterinary Medicine, Hokkaido University, Sapporo, Hokkaido 060-0818, Japan. ${ }^{\varpi}$ email: k-aoshima@vetmed.hokudai.ac.jp
} 
IL-10, and TGF- $\beta^{11-13}$. M1 macrophages are polarized by lipopolysaccharides (LPS) and interferon- $\gamma$ (IFN $\gamma$ ), while M2 macrophages are promoted by IL-4, IL-10 and TGF $\beta^{11-13}$. To distinguish M2 macrophages from M1 macrophages in tumor tissues, CD204 (also known as MSR1) has been used as a M2 macrophage marker ${ }^{14,15}$. Although LPS is an M1 macrophage inducer, LPS can also induce CD204 expression via the MAPK/ERK pathway in mouse macrophages ${ }^{16,17}$. It is highly likely that M2 macrophages also support tumor development and facilitate immune evasion in HSA; however, there are no studies that have evaluated their presence and functions in HSA.

In this study, we aimed to evaluate existing mouse HSA cell lines and immortalized endothelial cells for their possible use as a syngeneic model for canine HSA and to identify the constituents of the tumor microenvironment and their roles in canine and mouse HSA.

\section{Results}

ISOS-1 cells can be used as a syngenic model of canine HSA. To find syngeneic models for canine HSA, we first characterized two mouse endothelial tumor cell lines: ISOS-1 and UV +2 , and one immortalized mouse endothelial cell line, LEII. Morphologically, in single layer culture, both the mouse and canine HSA cell lines are characterized by spindle to polygonal cells with a moderate amount of cytoplasm and elongated nuclei arranged in cobblestone pattern (Fig. S1). Then, we inoculated ISOS-1, LEII and UV $2_{2}$ into Balb/c mice subcutaneously to determine their tumorigenic potential. All mice inoculated with ISOS-1 cells developed tumors after 30 days post inoculation (dpi) and reached the endpoint before 65 dpi (Fig. S1). Mice inoculated with $\mathrm{UV}+2$ or LEII cells, however, did not develop tumors. No metastasis was observed in ISOS-1 inoculated mice at the endpoint. We then made histopathological sections of ISOS-1 tumors and compared their morphologies with canine clinical HSA cases. ISOS-1 tumor cells formed variably sized, irregular shaped blood vessels that were separated by thin septa and trabeculae. They showed solid and capillary growth patterns which were also observed in canine HSA (Fig. 1A). Spindle-shaped neoplastic endothelial cells line luminal space in a single layer and are plump, hyperchromatic, and larger than normal endothelial cells (Fig. 1A, insets).

Next, we investigated whether ISOS-1, LEII and UV으 2 have similar molecular characteristics with canine HSA. Although $K d m 1 a, K d m 2 a$ and $K d m 2 b$ gene expressions in the mouse endothelial cell lines were not expressed more than twofold compared with primary mouse lung endothelial cells (MLEC), their protein expressions in ISOS-1 were significantly higher than in MLEC, LEII and UV 2 (Fig. 1B and Fig. S2). ISOS-1 tumor cells in Balb/c mice also expressed KDM2B as high as in canine HSA (Fig. 1C). Furthermore, ISOS-1 cells had other similar molecular features to canine HSA cell lines such as cellular aneuploidy, low p-ERK expression level and high c-FOS, $\gamma$ H2A.X and H2AK119Ub1 expression levels (Fig. 1D-G and Fig. S2).

Finally, we treated the mouse endothelial cell lines with GSK-J4, a histone demethylase inhibitor, to assess whether GSK-J4 can inhibit their viability and whether it is more effective than doxorubicin like in canine HSA. The results showed that GSK-J4 could inhibit the cell viability of ISOS-1, LEII and UVO 2 at a lower IC In $_{50}$ value compared to doxorubicin (Fig. S3).

These results demonstrate similarities between ISOS- 1 cell line and canine HSA and suggest that ISOS- 1 can be used as a syngeneic model for HSA.

$\mathrm{CD} \mathrm{O4}^{+}$macrophages are the major constituent in HSA tumor microenvironment. To identify the constituent cells of the tumor microenvironment in HSA, we immunohistochemically stained four ISOS-1 tumors developed in Balb/c mice and twenty-eight clinical HSA samples in dogs with antibodies for Ibal and CD3, a macrophage and a T cell marker, respectively. T cells were mostly confined in the periphery of tumor tissues in ISOS-1 tumors, and the number of T cells and their distribution were variable in canine clinical HSA samples. However, Iba1 staining revealed that macrophages were the major components of tumor microenvironment in both ISOS-1 tumor and canine HSA cases (Fig. 2A). The average percentages of macrophages in tumor tissues were $65.27 \%$ and $48.83 \%$ in ISOS- 1 tumors and canine HSA cases, respectively (Fig. 2B).

Next, to assess whether HSA cells actively recruit macrophages, we performed cell migration assay and compared the number of migrated macrophage-like RAW264.7 cells in conditioned media from MLEC or ISOS-1. As a result, the number of migrated RAW264.7 cells significantly increased when cultured in conditioned medium from ISOS-1 compared to the one from MLEC (Fig. 2C,D). RAW264.7 cells cultured in ISOS-1 conditioned medium did not induce M1 macrophage-related genes but highly expressed M2 macrophage markers such as HAVCR2 and CD163 (Fig. 2E). The protein expression of CD204, another M2 macrophage marker, was also induced by ISOS-1 conditioned medium in RAW264.7 cells (Fig. 2F, Fig. S4). Lastly, we stained ISOS-1 tumors and canine HSA cases with anti-CD204 antibody and identified a large number of CD204 positive cells in ISOS-1 tumors and canine HSA samples (Fig. 2G).

These results suggest that HSA tumor cells can recruit macrophages into the tumor microenvironment and polarize them to M2 macrophages.

Tumor cells and tumor infiltrating macrophages express PD-L1 in HSA. In both ISOS-1 tumor and canine HSA cases, macrophages dominate the tumor parenchyma along with the tumor cells, thus, it is important to learn how these macrophages contribute to HSA pathogenesis. CD204 $4^{+}$macrophages in tumor tissues have been reported to express PD-L1, and they are associated with tumor malignancy and PD-L1 upregulation in tumor cells and immune cells ${ }^{14,15}$. We, therefore, examined PD-L1 expressions in tumor cells and macrophages in ISOS-1 tumors, clinical canine HSA cases and HSA cell lines.

First, we performed immunohistochemistry for PD-L1 on ISOS-1 tumor tissues developed in Balb/c mice and canine HSA cases. We found that PD-L1 signals were detected weakly in tumor cells and strongly in tumor infiltrating macrophages (Fig. 3A). Double staining of PD-L1 and Iba1verified that macrophages in ISOS-1 tumors and canine HSA cases expressed PD-L1 (Fig. 3B). In canine clinical HSA cases, 11 cases (53.6\%) showed 
A ISOS-1
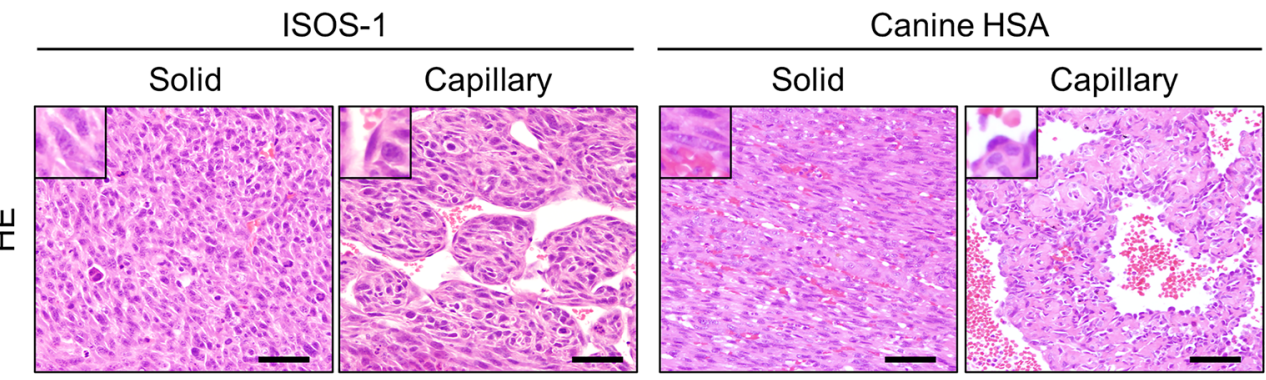

B

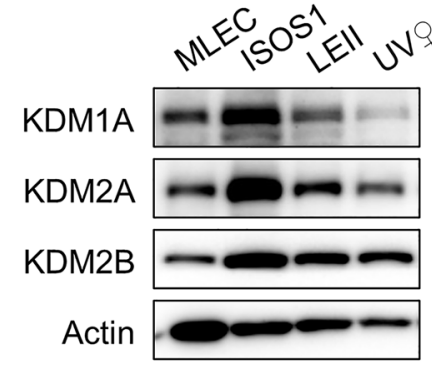

C

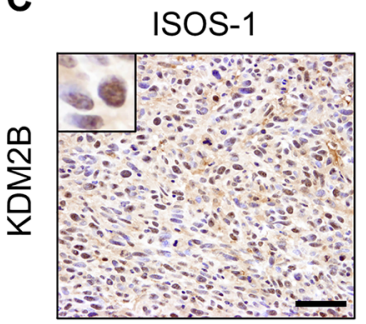

Canine HSA

D
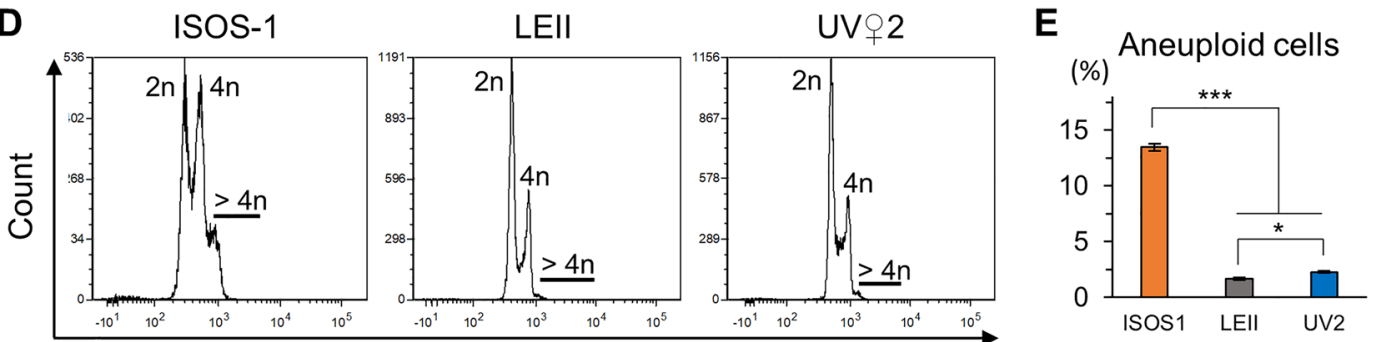

Propidium iodide

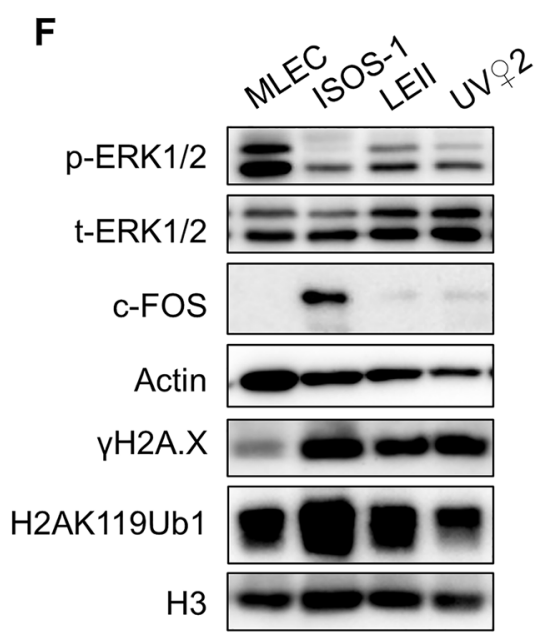

G
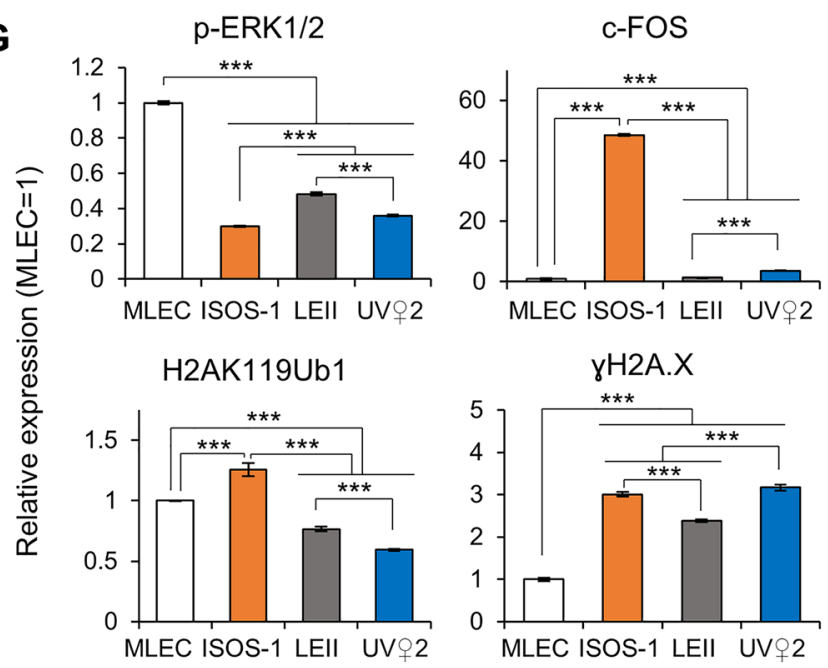

Figure 1. ISOS-1 presents similar morphology and molecular features with canine HSA. (A) Hematoxylin and eosin (HE) staining in ISOS-1 and canine HSA tumors. (B) KDM1A, KDM2A, and KDM2B protein expressions in MLEC, ISOS1, LEII, and UV 2 cell lines. (C) KDM2B immunohistochemistry of ISOS-1 and canine HSA tumors. (D) Histograms of PI intensities in ISOS1, LEII, and UV 2 cell lines. (E) Percentages of cells with aneuploidy in ISOS-1, LEII, and UV 92 cell lines. (F) Western blotting for phosphorylated ERK1/2 (p-ERK1/2), c-FOS, $\gamma$ H2A.X and H2AK119ub1 in MLEC, ISOS-1, LEII, and UV 2 cell lines. (G) Quantification of p-ERK1/2, c-FOS, $\gamma \mathrm{H} 2 \mathrm{~A} . \mathrm{X}$, and H2AK119ub1 in MLEC, ISOS-1, LEII, and UV 2 cell lines. p-ERK1/2 expression was normalized with total ERK1/2 expression. c-FOS expression was normalized with Actin expression. H2AK119Ub1 and $\gamma \mathrm{H} 2 \mathrm{~A}$.X expressions were normalized with $\mathrm{H} 3$ expression levels. The protein expression levels in MLEC were set to 1 . Data are presented as mean values \pm s.d. Experiments were performed in triplicates. Scale $=125 \mu \mathrm{m} .{ }^{* *} P<0.001$, Tukey's test. Uncropped western blotting data is found in Supplementary Fig. S2. 
A
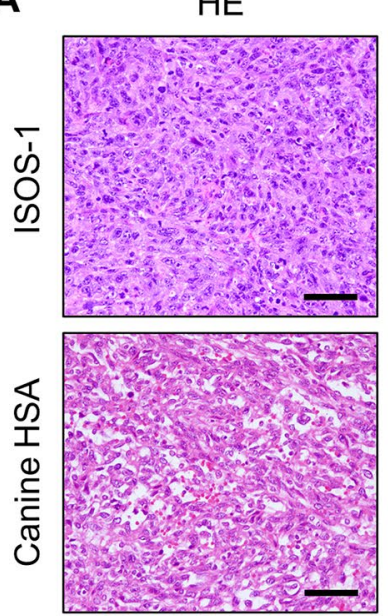

C

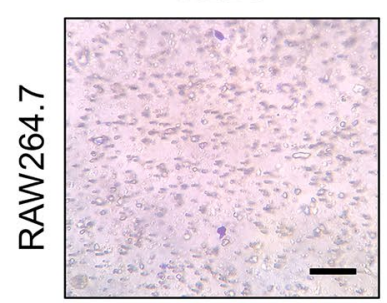

CD3
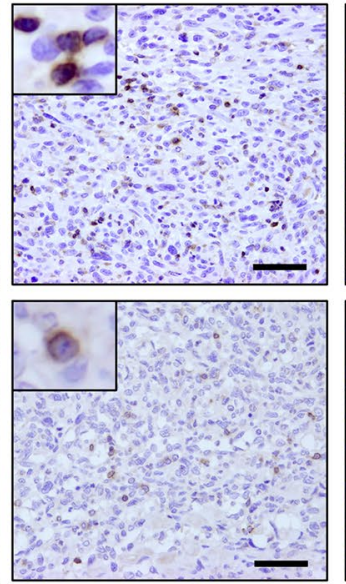

ISOS-1

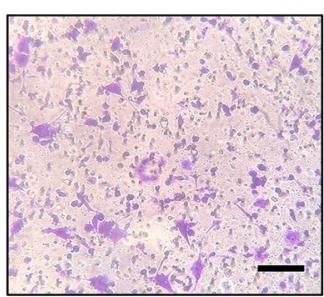

Iba1
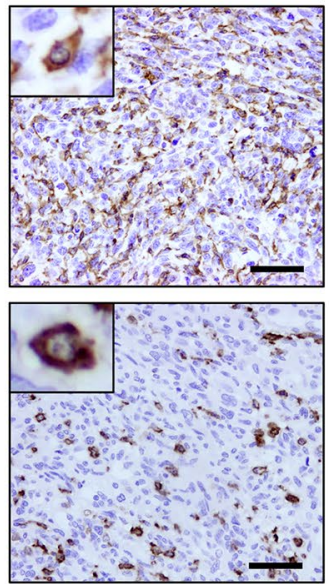

B

Iba1 positive cells

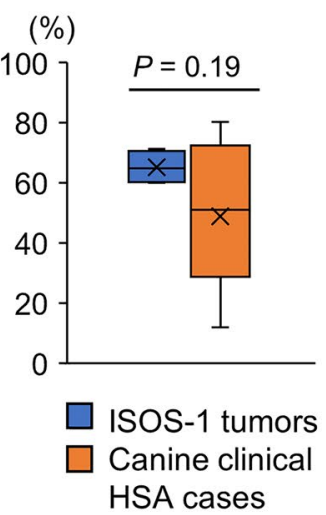

E

D
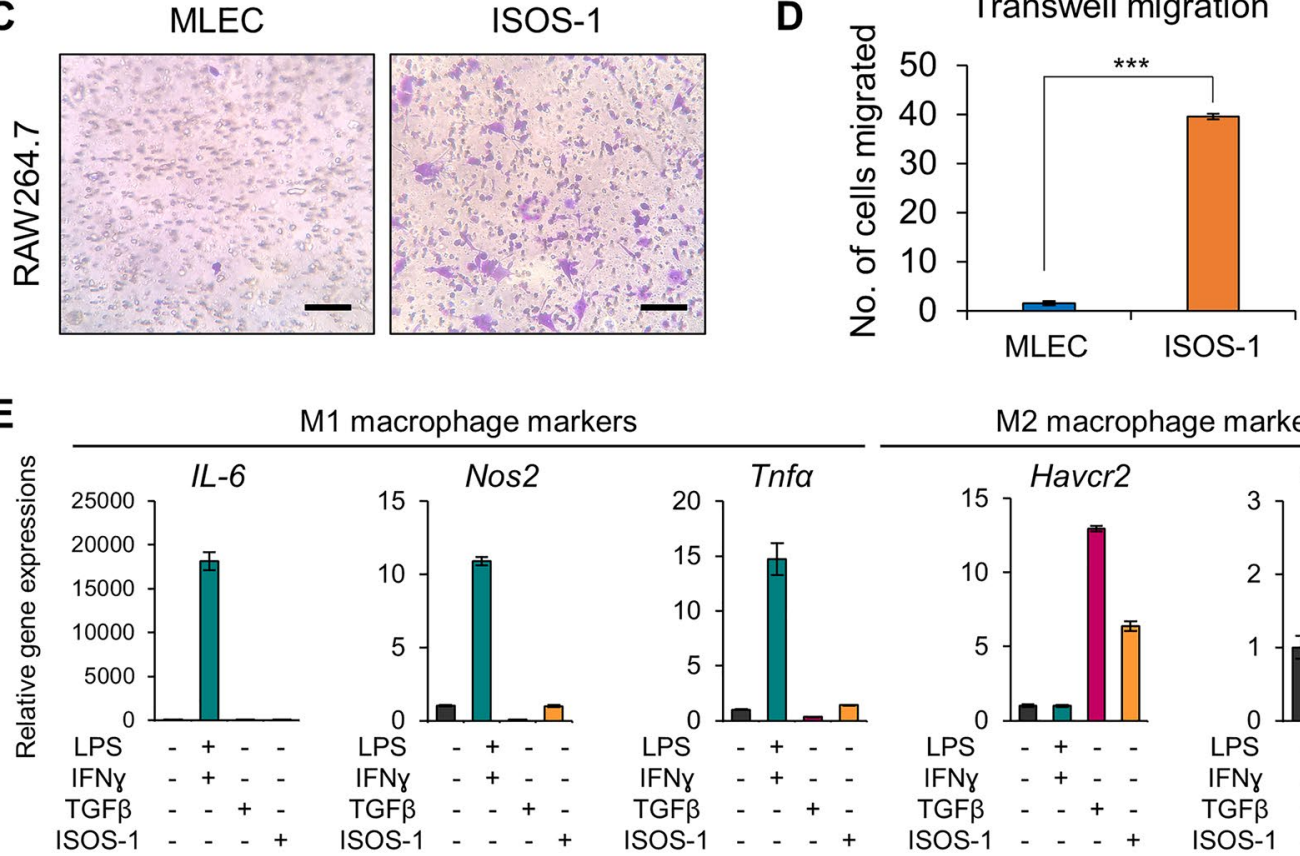

M1 macrophage markers

M2 macrophage markers
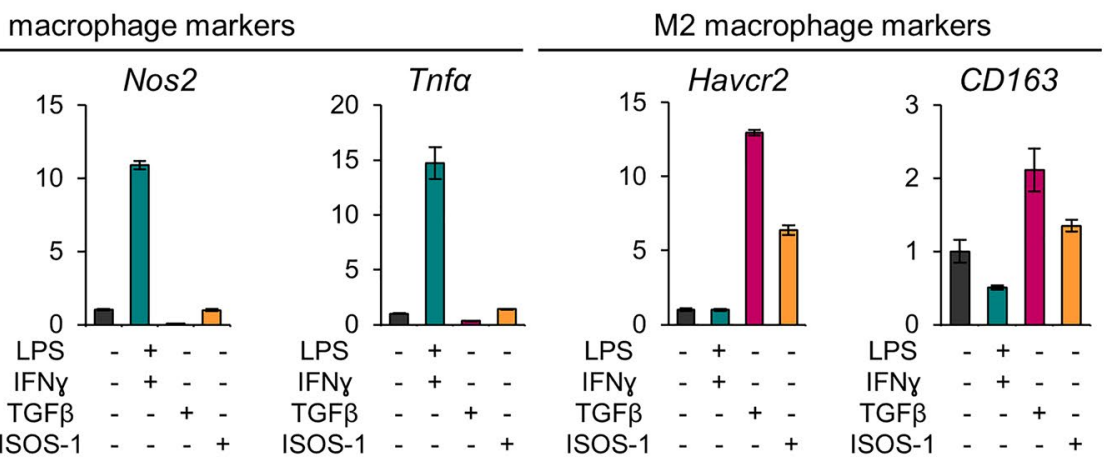

$\mathbf{F}$
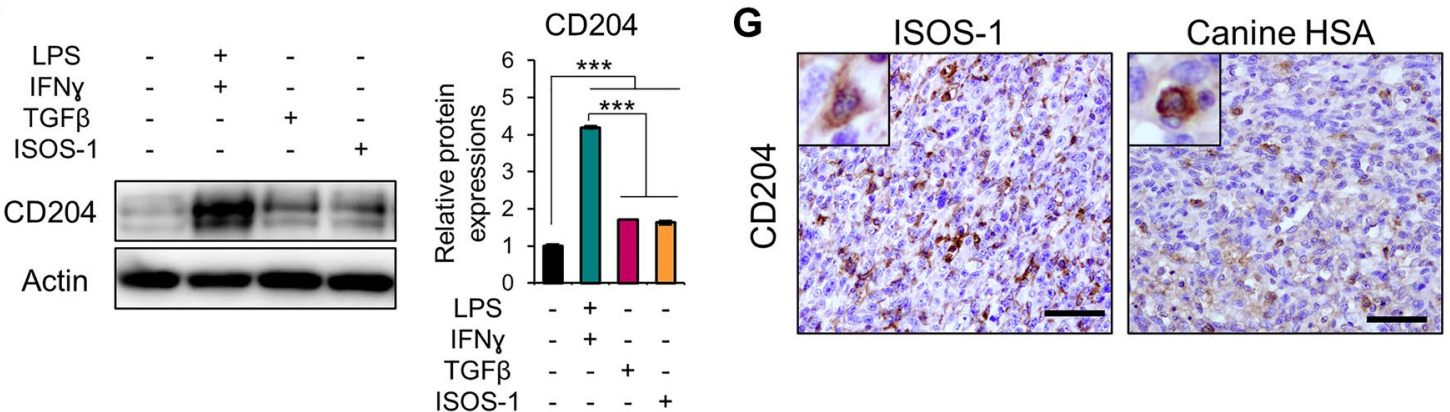

Figure 2. HSA cells attract macrophages and polarize them to M2 macrophages. (A) HE staining and immunohistochemistry of CD3 and Iba1 for ISOS-1 and canine HSA tumors. (B) Quantitative analysis of Iba1 positive cells in ISOS-1 and canine HSA tumors. Y-axis indicates the percentages of Ibal positive cells relative to all cells comprising the tumor tissue. (C) Cell migration assay in RAW264.7 cells cultured over MLEC- or ISOS-1-conditioned media. (D) Quantitative analysis of (C). (E) Gene expressions of M1 or M2 macrophage markers in untreated, LPS and IFN-treated, TGF $\beta$-treated, and ISOS- 1 conditioned media-treated RAW264.7 cells. (F) (Left) Western blot analysis for CD204 in untreated, LPS and IFN-treated, TGF $\beta$-treated, and ISOS1 conditioned media-treated RAW264.7 cells. (Right) Quantitative analysis of the western blotting data. (G) Immunohistochemistry of CD204 for ISOS-1 and canine HSA tumors. Scale $=125 \mu \mathrm{m}$. Data are presented as mean values \pm s.d. 
A
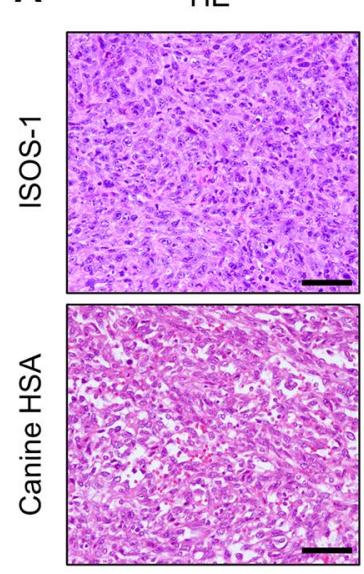

B

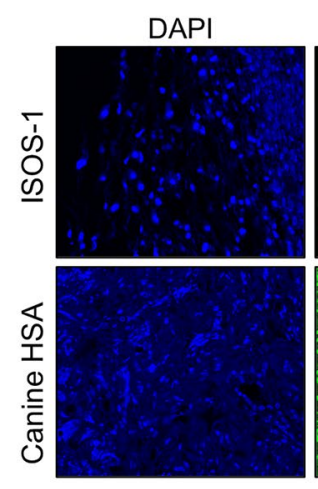

PD-L1

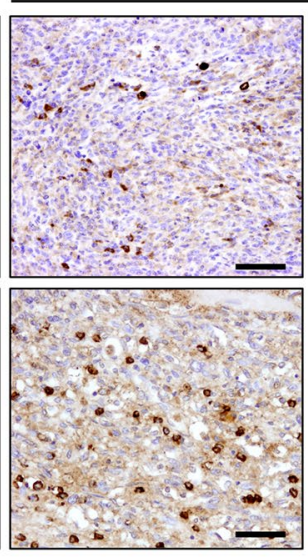

PD-L1

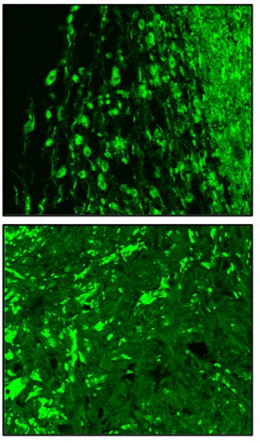

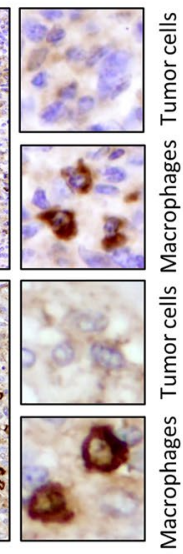

Iba1

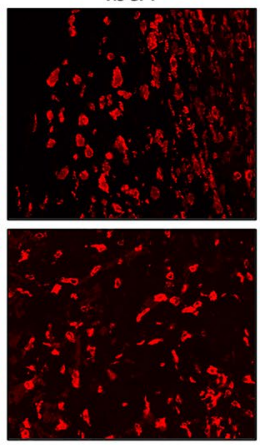

C

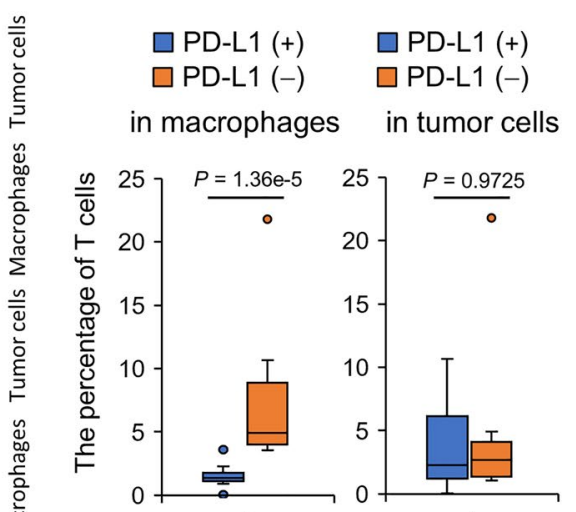

Figure 3. HSA tumor cells and tumor infiltrating macrophages express PD-L1. (A) HE staining and immunohistochemistry of PD-L1 for ISOS-1 and canine HSA tumors. HE staining images are the same as in Fig. 2A because the same samples were used for this experiment. (B) Immunofluorescence assay for PD-L1 and Iba1 in ISOS-1 and canine HSA tumors. Arrows indicate the cells expressing both PD-L1 and Iba1. (C) The percentages of T cells infiltrating in canine HSA tumor tissues with or without PD-L1 expressions in macrophages or tumor cells. (D) Representative T cell staining images of canine HSA tumor tissues with or without PD-L1 expressions in macrophages. Scale $=125 \mu \mathrm{m}$. $P$-values were calculated by Mann-Whitney $U$ test.

PD-L1 signals in both tumor cells and macrophages, whereas 4 and 8 cases had PD-L1 expressions in either tumor cells or macrophages, respectively (Table 1). To address whether PD-L1 expressions affect tumor immune responses, we evaluated $T$ cell infiltration in tumor tissues of clinical canine HSA cases by CD3 staining. The results demonstrated that the percentages of T cells in tumor tissues with PD-L1 expressions in macrophages were significantly lower than the cases without PD-L1 expression in macrophages (Fig. 3C,D). PD-L1 expression in tumor cells did not significantly affect the number of T cells in tumor tissues (Fig. 3C).

In in vitro settings, PD-L1 also expressed in ISOS-1 and canine HSA cell lines: JuB2, JuB4 and Re21 (Fig. 4A,B, Fig. S5). The number of PD-L1 positive cells was slightly increased by IFN $\gamma$ treatment in ISOS-1, whereas almost $100 \%$ of canine HSA cells expressed PD-L1 without IFN $\gamma$ treatment (Fig. 4A,B, Fig. S5). Then, we tested whether HSA tumor cells can induce PD-L1 expression in macrophages using RAW264.7 cells. PD-L1 gene expression in RAW264.7 cells was induced by LPS and IFN $\gamma$ treatment (Fig. 4C). Furthermore, ISOS-1 conditioned medium significantly induced PD-L1 protein expression in RAW264.7 cells (Fig. 4D,E). We further confirmed that ISOS-1 conditioned medium could induce PD-L1 expression in mouse peritoneal macrophages (Fig. 4F,G).

These results suggest that HSA can evade immune attack through PD-L1 expression in tumor cells and by inducing PD-L1 expression in macrophages.

\section{Discussion}

Here we demonstrated that a mouse HSA cell line, ISOS-1, can be used as a syngenic model for HSA, and that macrophages are the major constituent of the HSA tumor microenvironment in both ISOS- 1 tumors and canine clinical HSA cases. In this study, we used two mouse HSA cell lines (ISOS-1 and UV 2 ) and one immortalized mouse endothelial cell line (LEII) as candidates of syngenic models for canine HSA. As previous studies reported, ISOS- 1 was the only mouse HSA cell line that developed tumors in immunocompetent mice ${ }^{9,18}$. UV $\bigcirc 2$ and LEII might be rejected by host immune responses after inoculation. ISOS-1 possessed similar molecular features with 


\begin{tabular}{|c|c|c|c|c|c|c|}
\hline Patient no. & Sex & Age (years) & Breed & $\begin{array}{l}\text { PD-L1 intensity } \\
\text { (tumor cells) }\end{array}$ & $\begin{array}{l}\text { PD-L1 intensity } \\
\text { (macrophages) }\end{array}$ & $\begin{array}{l}\text { PD-L1 intensity } \\
\text { (lymphocytes) }\end{array}$ \\
\hline 1 & Male (castrated) & 7 & $\begin{array}{l}\text { Miniature Dachs- } \\
\text { hund }\end{array}$ & - & +++ & - \\
\hline 2 & Female (spayed) & 7 & Golden Retriever & + & - & - \\
\hline 3 & Male & 14 & Miniature Schnauzer & - & - & - \\
\hline 4 & Female (spayed) & 10 & Mixed & + & +++ & - \\
\hline 5 & Male (castrated) & 12 & Beagle & + & - & - \\
\hline 6 & Male & 8 & Labrador Retriever & ++ & +++ & - \\
\hline 7 & Female (spayed) & 10 & $\begin{array}{l}\text { Miniature Dachs- } \\
\text { hund }\end{array}$ & ++ & +++ & - \\
\hline 8 & Female (spayed) & 14 & $\begin{array}{l}\text { Miniature Dachs- } \\
\text { hund }\end{array}$ & - & +++ & - \\
\hline 9 & Female (spayed) & 14 & Miniature Schnauzer & - & - & - \\
\hline 10 & Male (castrated) & 12 & Miniature Schnauzer & + & +++ & - \\
\hline 11 & Male & 13 & French Bulldog & - & - & - \\
\hline 12 & Male (castrated) & 14 & $\begin{array}{l}\text { Miniature Dachs- } \\
\text { hund }\end{array}$ & - & +++ & - \\
\hline 13 & Female (spayed) & 12 & Labrador Retriever & - & +++ & - \\
\hline 14 & Male (castrated) & 14 & Lhasa Apso & + & - & - \\
\hline 15 & Female (spayed) & 12 & Beagle & - & +++ & - \\
\hline 16 & Female (spayed) & 14 & $\begin{array}{l}\text { Miniature Dachs- } \\
\text { hund }\end{array}$ & + & +++ & - \\
\hline 17 & Male (castrated) & 14 & $\begin{array}{l}\text { Miniature Dachs- } \\
\text { hund }\end{array}$ & ++ & +++ & - \\
\hline 18 & Female & 8 & Flat-coated Retriever & - & +++ & - \\
\hline 19 & Female (spayed) & 11 & Scottish Terrier & ++ & +++ & - \\
\hline 20 & Female & 5 & Golden Retriever & ++ & +++ & - \\
\hline 21 & Male & 5 & Beagle & ++ & +++ & - \\
\hline 22 & Female & 9 & Golden Retriever & + & - & - \\
\hline 23 & Male & 10 & Miniature Schnauzer & ++ & +++ & - \\
\hline 24 & Female & 12 & Mixed & - & - & - \\
\hline 25 & Male & 9 & Great Pyrenees & - & +++ & - \\
\hline 26 & Male & 7 & Jack Russel Terrier & - & - & - \\
\hline 27 & Male (castrated) & 8 & Labrador Retriever & - & +++ & - \\
\hline 28 & Male (castrated) & 10 & Maltese & + & +++ & - \\
\hline
\end{tabular}

Table 1. PD-L1 expression in tumor and immune cells in canine HSA.

canine HSA such as high KDM2B expression and ubiquitination of its target H2AK119, whereas high KDM2B expression and high H2AK119ub1 level were not detected in UV +2 and LEII. ISOS-1 cells were aneuploid, which implies that they have severe DNA stresses leading to apoptotic cell death ${ }^{19}$. ISOS- 1 cells probably overcome cell deaths by deactivating a pro-apoptotic protein ERK1/2 and activating DNA repair proteins such as c-FOS and $\gamma \mathrm{H} 2 \mathrm{~A}$.X. Given these results and our previous findings that KDM2B plays an important role in canine HSA, KDM2B is probably a common factor for endothelial cell tumor malignancy.

We also showed that ISOS-1 cells recruited macrophages, polarized them to M2 macrophages, and induced them to express PD-L1. In canine HSA tumors, macrophages expressed both CD204 ${ }^{+}$and PD-L1, which suggests that canine HSA cells also attract and induce macrophages to express PD-L1. Since a smaller number of T cells infiltrated in tumor tissues of canine clinical HSA cases with PD-L1 positive macrophages, macrophages could facilitate immune evasion through induction of PD-L1 expression in canine HSA. Antibodies specific for canine PD-L1 have been developed and tested for their safety and efficacy in canine tumor patients although anti-PDL1 antibody treatment has not been studied in canine HSA patients ${ }^{20-22}$. Given that almost $70 \%$ of clinical HSA cases examined in our study expressed PD-L1 in macrophages, immunotherapy using anti PD-L1 antibody treatment could be useful as an alternative treatment for canine HSA. Furthermore, in our previous study, silencing of KDM2B resulted to increased interferon gamma and alpha responses ${ }^{7}$. This implies that KDM2B inhibition induces immune reactions; therefore, combination therapy with anti-PD-L1 antibody and KDM2B inhibition might provide better outcomes than a single treatment with anti-PD-L1 or KDM2B inhibitor.

In summary, we identified similarities between ISOS-1 and canine HSA, and we demonstrated the usefulness of ISOS-1 as a syngeneic model for canine HSA. By taking advantage of ISOS-1 cells, we characterized the tumor microenvironment in HSA and demonstrated the crosstalk between tumor cells and macrophages for the induction of PD-L1 expression. These results provide useful insights for understanding HSA pathology and will be beneficial to develop novel therapeutics for HSA. 

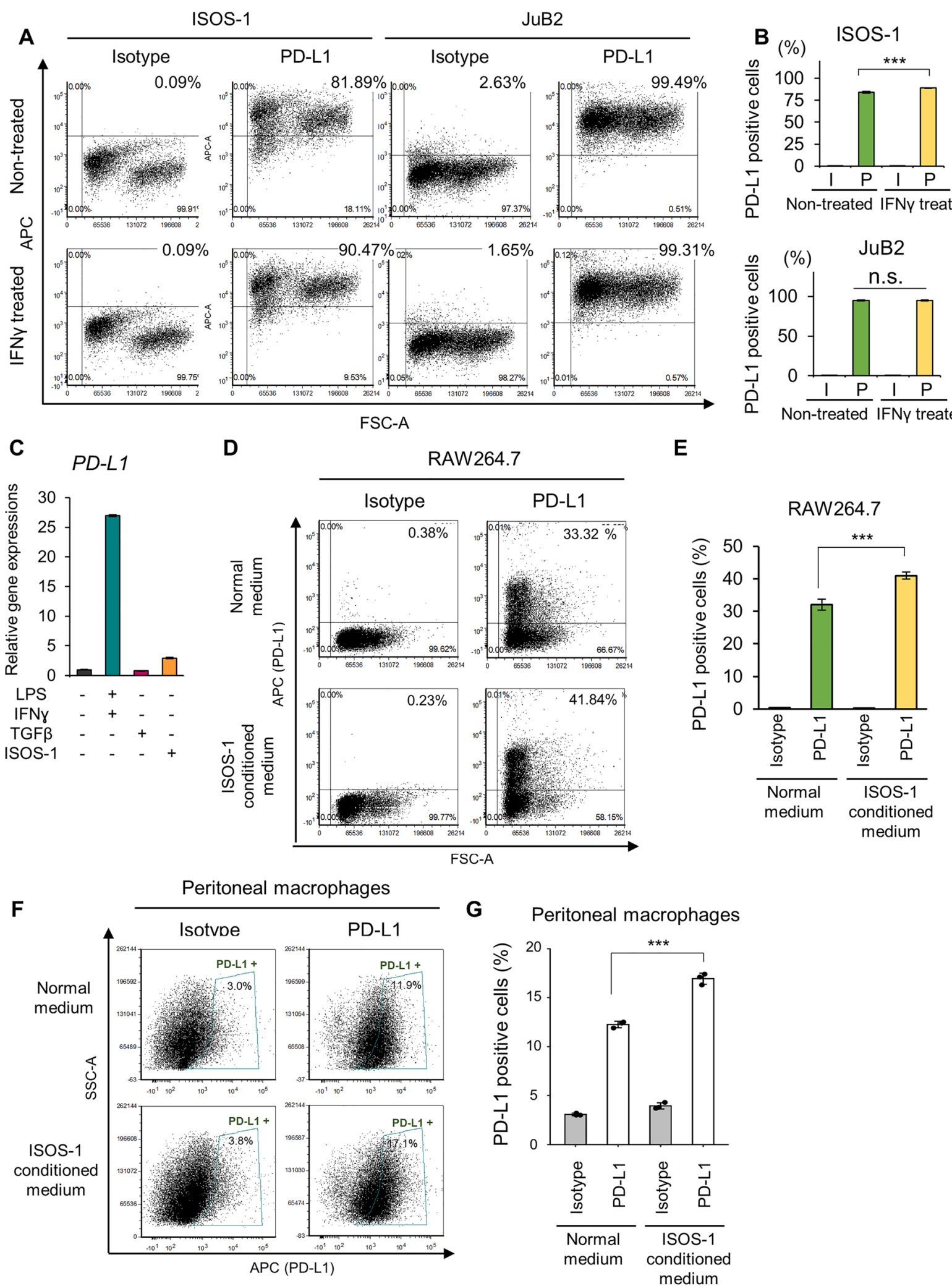

G Peritoneal macrophages

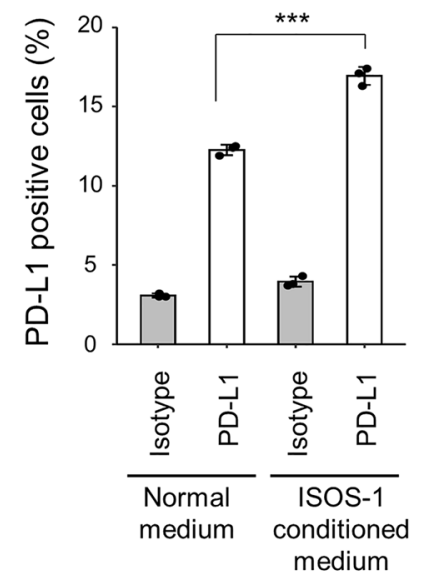

Figure 4. ISOS-1 cells induce PD-L1 expression in RAW264.7 cells. (A) Representative images of flow cytometry analysis for PD-L1 in ISOS-1 and JuB2 cell lines with/without IFN $\gamma$ treatment. APC indicates PD-L1 expressions. (B) Quantitative analysis of A. (C) Gene expression levels of PD-L1 in untreated, LPS and IFNtreated, TGF $\beta$-treated, and ISOS-1 conditioned media-treated RAW264.7 cells. (D) Representative images of flow cytometry analysis for PD-L1 in RAW264.7 cells cultured in normal or ISOS-1 conditioned media. (D) Quantitative analysis of (C). (E) Representative images of flow cytometry analysis for PD-L1 in mouse peritoneal macrophages cultured in normal or ISOS-1 conditioned media. (F) Quantitative analysis of (E). Data are presented as mean values \pm s.d. ${ }^{\star * \star} P<0.001$, Tukey's test. 


\section{Materials and methods}

This study was approved by the Animal ethical committee of Hokkaido University (20-0083). All methods and protocols were performed in accordance with Hokkaido University guidelines.

Cell lines. ISOS-1 cells were obtained from the Cell Resource Center for Biomedical Research Cell Bank (Tohoku University) ${ }^{9}$. UV +2 cells were obtained from RIKEN Bioresource Center ${ }^{10}$. The LEII cell line was donated by Dr. Kazuhiro Kimura (Hokkaido University) and cultured as described previously ${ }^{23,24}$. RAW264.7 cells were obtained from RIKEN Bioresource Center ${ }^{25}$. Canine HSA cell lines (JuB2, JuB4, Re12, Ud6) were given by Dr. Hiroki Sakai (Gifu University) ${ }^{26}$. All cells used in this study were routinely tested for Mycoplasma using PCR and were submitted to ICLAS Monitoring Center (Kawasaki, Japan) for Mouse hepatitis virus testing ${ }^{27,28}$.

Mouse lung endothelial cell isolation. The primary mouse lung endothelial cells (MLEC) were isolated from a 10-week-old, female, Balb/c mice and were cultured as described elsewhere ${ }^{29}$. Briefly, freshly isolated mouse lung was minced using autoclaved scissors, digested by collagenase I, and filtered through a $70-\mu \mathrm{m}$ cell strainer. The cell suspension was incubated with anti-rat Dynabeads (Thermo Fisher Scientific, MA, USA, 11035) conjugated with anti-mouse CD31 antibody (BD Biosciences, NJ, USA, 557355). Pooled cells were seeded in a 12 -well-plate pre-coated with $0.1 \%$ gelatin. Upon reaching confluence, the cells were trypsinized and then incubated with anti-mouse ICAM-2 antibody (BD Biosciences, NJ, USA, 553326) conjugated Dynabeads. Pooled cells were seeded in 12 -well-plates pre-coated with $0.1 \%$ gelatin. Harvested cells were assessed using tube formation assay, 1,1'-dioctadecyl-3,3,3',3'-tetramethyl-indocarbocyanine perchlorate Low Density Lipoprotein (DiI-Ac-LDL) uptake, and CD31 gene expression (Fig. S4).

Tube formation assay. Tube formation was performed as described previously ${ }^{30}$. Briefly, $1 \times 10^{5} \mathrm{JuB} 2$ or MLEC suspended in 24-h JuB2 conditioned medium were seeded in a 24 -well plate pre-coated with Corning ${ }^{\circ}$ Matrigel $^{\circ}$ Basement Membrane Matrix (Corning Inc. NY, USA). Cells were observed at 0, 2, 4, and $8 \mathrm{~h}$ after seeding for tube formation.

Dil-Ac-LDL uptake assay. Dil-Ac-LDL uptake assay was performed with Dil-Ac-LDL staining kit (Cell Applications, Inc., $022 \mathrm{~K}$ ) according to the manufacturer's instructions. Briefly, $6 \times 10^{5}$ of isolated MLEC were cultured in a 4-well chamber slide (Nunc Lab-Tek Chamber Slide System, Thermo Fisher Scientific) precoated with Extracellular Matrix Attachment Solution provided in the kit. MLEC were allowed to grow until 95\% confluency. Culture medium from each chamber was removed and cells were cultured in $100 \mu \mathrm{L}$ of culture medium supplemented with $10 \mu \mathrm{g} / \mathrm{mL}$ of Dil-Ac-LDL. Cells were incubated for $4 \mathrm{~h}$, washed with wash buffer, and then mounted with DAPI-containing mounting medium and covered with $22 \times 50 \mathrm{~mm}$ coverslip. Cells were examined under a confocal microscope (LSM700, Carl Zwiss, Oberkochen, German).

Mice. All mouse experimental protocols were approved by the institutional committee of animal care and use in Hokkaido University (protocol number: 20-0083) and were performed in compliance with ARRIVE guidelines. Six-week-old male and female Balb/c mice purchased from Japan SLC, Inc. (Shizuoka, Japan) were used as breeders for tumor transplantation experiments. Mice were kept in a temperature-controlled specific-pathogenfree facility with a $12 \mathrm{~h}$ light/dark cycle. Animals in all experimental groups were examined at least twice weekly for tumorigenesis.

Tumor transplantation studies. ISOS-1, LEII, and UV $q 2$ cell lines were cultured in $15 \mathrm{~cm}$ dishes accordingly. Mice were randomly assigned to each group. $2 \times 10^{6}$ ISOS-1, LEII, or UV 2 cells were resuspended in Corning Matrigel $^{\circ}$ Basement Membrane Matrix (Corning Inc. NY, USA, 649-54961) and inoculated subcutaneously in mice anesthetized with $3 \%$ isoflurane. Tumor sizes were measured twice weekly one week after inoculation. Mice were euthanized with $\mathrm{CO}_{2}$ when tumors reached $1500 \mathrm{~mm}^{3}$ in volume or when mice exhibited abnormal behavior. Tumors were fixed in $10 \%$ neutral buffered formalin and processed for routine histological examination.

Cell viability analysis. Cell viability after doxorubicin or GSK-J4 treatment was measured with Cell Counting Kit-8 (Dojindo Molecular Technologies, Inc., Kumamoto, Japan, 343-07623) according to the manufacturer's instructions. The absorbance at $450 \mathrm{~nm}$ was measured with NanoDrop ${ }^{\mathrm{Tm}} 2000$ (Thermo Fisher Scientific). Determination of $\mathrm{IC}_{50}$ were performed using KyPlot 6.0 software (KyensLab, Inc., Tokyo, Japan). Experiments were performed at least three times with triplicates.

Western blotting. Western blotting was performed as described previously ${ }^{7}$. The antibodies used in this study are as follows: anti-KDM1A antibody (1:1000; Cell Signaling Technology, MA, USA, 2139S), anti-KDM2A antibody (1:1000; Abcam, Cambridge, UK, Ab191387), anti-KDM2B antibody (1:1000; Santa Cruz Biotechnology, Inc., TX, USA, sc-293279), anti Actin antibody (1:10000; Sigma Aldrich, MO, MAB1501), anti-c-FOS antibody (1:1000; Santa Cruz Biotechnology, Inc., sc-166940), anti- $\gamma$ H2A.X antibody (1:1000; Bethyl Laboratories, Inc, TX, USA. A300-081A-T), anti-p-ERK1/2 antibody (1:1000; Cell Signaling Technology, 4370S), antiERK1/2 antibody (1:1000; Cell Signaling Technology, 4695S), anti-H2AK119Ub1 antibody (1:2000; Cell Signaling Technology,8240S), anti-H3 antibody (1:3000; MAB Institute, Inc. Yokohama, Japan, MABI0001-20), and CD204 (1:125; Medicinal Chemistry Pharmaceutical Co., Ltd., Sapporo, Japan, KT022). All primary antibodies were diluted in Can Get Signal Solution 1 (TOYOBO, Osaka, Japan, NKB-201). Membranes were washed with 
Tris-Buffered Saline with $0.1 \%$ Tween $^{\circ} 20$ (TBS-T) three times for 5 min each time before incubating with ECL Mouse IgG HRP-linked whole antibody (Cytiva, MA, USA, \#NA934) or ECL Rabbit IgG HRP-linked whole antibody (Cytiva, \#NA931) diluted in Can Get Signal Solution 2 (TOYOBO, Osaka, Japan, NKB-301). Signal development was performed using Immobilon Western Chemiluminescent HRP substrate (Merck Millipore, NJ, USA, WBKLS0100). ImageQuant LAS 4000 mini luminescent image analyzer (GE Healthcare) was used to visualize chemiluminescent signals and the ImageJ software was used to process captured data ${ }^{31}$.

Quantitative RT-PCR (qRT-PCR). $\quad$ qRT-PCR was performed as described previously ${ }^{7}$. The list of primers used in this study is listed in Supplementary Table. cDNA of mouse mesenchymal stem cells (mMSC), donated by Dr. Yusuke Komatsu (Hokkaido University), were used as a negative control for CD31 expression.

Isolation of peritoneal macrophages. Five-week male Balb/c mice were injected with $3 \%$ thioglycolate broth intraperitoneally. Two days after the treatment, the peritoneal cavity was washed with PBS, and the PBS was collected and centrifuged. Cell pellets were resuspended with DMEM and $3 \times 10^{6}$ cells were seeded in 6 well plates and cultured in normal DMEM at $37^{\circ} \mathrm{C}$ in $5 \% \mathrm{CO}_{2}$ for $1 \mathrm{~h}$. Then, the cells were washed with PBS twice and cultured with either normal DMEM or $48 \mathrm{~h}$-conditioned DMEM from ISOS-1 cell culture. After $24 \mathrm{~h}$-culture, the cells were used as peritoneal macrophages for flow cytometry analysis.

Flow cytometry analysis. Flow cytometry for cell cycle was performed as described previously ${ }^{7}$. Briefly, $2 \times 10^{5} \mathrm{HSA}$ cells were harvested for each replicate and unstained control. Samples were fixed with $70 \%$ ethanol and incubated with propidium iodide (PI) in the dark for $30 \mathrm{~min}$ at $37^{\circ} \mathrm{C}$. Unstained cells were incubated with PBS for 30 min at $37^{\circ} \mathrm{C}$. Cell cycle was analyzed in BD FACSVerse ${ }^{\mathrm{ms}}$ flow cytometer (BD Biosciences, NJ, USA). Results were analyzed with FCS Express 4 software (De Novo Software, CA, USA). Experiments were performed at least three times with triplicates.

For PD-L1 expression analysis, HSA cells were cultured in 6-well plates until 90\% confluency. Cells were washed with $1.34 \mathrm{mM}$ EDTA in PBS twice and then detached by adding $1 \mathrm{~mL}$ of $1.34 \mathrm{mM}$ EDTA solution and incubating at room temperature (RT) for 10-15 min. Cells were counted and $2 \times 10^{5}$ cells were used for each replicate. Cells were blocked with 10\% goat serum (Thermo Fisher Scientific, 16210-072) in PBS with sodium azide at RT for 15 min before incubating with anti PD-L1 antibody (1:100; clone 6C11-3A11) ${ }^{21}$ or isotype rat IgG2a control (1:50; BD Biosciences, 553927) at RT for $30 \mathrm{~min}$. Cells were washed with 1\% BSA in PBS twice before incubating with secondary anti-rat IgG antibody conjugated with APC (1:500; Southern Biotech, AL, USA, 3010-11L). Peritoneal macrophages were detached by adding $10 \%$ goat serum in PBS and scraping with a cell scraper. After PD-L1 staining was done as described above, cells were stained with FITC-conjugated anti-F4/80 antibody (1:200; Thermo Fisher Scientific, 11-4801-82) at RT for $30 \mathrm{~min}$, and then 7-AAD (BD Biosciences, 559925) was added before analyzing. Cells were analyzed in BD FACSVerse ${ }^{\text {Tw }}$ flow cytometer (BD Biosciences, NJ, USA). Results were analyzed with FCS Express 4 software (De Novo Software, CA, USA). To analyze the live macrophage population, the 7 -AAD negative and F4/80 positive population was gated and used for further analysis (Fig. S6). Experiments were performed at least three times with triplicates.

RAW264.7 polarization. RAW264.7 cells were polarized to M1 or M2 macrophages as described by a previous study ${ }^{32} .1 \times 10^{6}$ RAW264.7 cells were seeded in $10 \mathrm{~cm}$ dishes and were stimulated with LPS (100 ng/ $\mathrm{mL})$ and IFN- $\gamma(20 \mathrm{ng} / \mathrm{mL})$ or TGF- $\beta(10 \mathrm{ng} / \mathrm{mL})$ for $24 \mathrm{~h}$ for their polarization towards the M1 or M2 subtype, respectively. RAW264.7 cells were also incubated in $48 \mathrm{~h}$-conditioned medium from ISOS-1 cell culture filtered with a $0.45 \mu \mathrm{m}$ sterile filter. RAW264.7 cells cultured under normal DMEM medium were considered as M0 or unpolarized macrophages.

Cell migration assay. Cell migration assay for RAW264.7 cells was performed as described previously with minor changes ${ }^{33}$. Briefly, $2.5 \times 10^{5}$ RAW264.7 cells in $1 \mathrm{~mL}$ normal medium were added to ThinCerts cell culture inserts (Greiner Bio-One, Kremsmünster, Austria, 657630) in 6 well plates while $2.5 \mathrm{~mL}$ of $48 \mathrm{~h}$ conditioned medium from MLEC or ISOS-1 cells were added to the lower wells. After $24 \mathrm{~h}$, cells in the upper wells were removed with a cotton swab, the filters were fixed with $4 \%$ paraformaldehyde in PBS and stained with $0.1 \%$ crystal violet solution in DW. Cell migration was assessed by counting the number of migrated cells in five fields per well at $40 \times$ magnification.

Histopathological analysis and Immunohistochemistry. Tissue samples were fixed for at least $48 \mathrm{~h}$ in $10 \%$ neutral buffered formalin, processed routinely, embedded in paraffin, sectioned $3 \mu \mathrm{m}$ thick, mounted on glass slides, and stained with hematoxylin and eosin (HE). All tumors were examined for KDM2B expression and the presence of lymphocytes and macrophages using immunohistochemistry (IHC). IHC was performed using antibodies to Iba1 (1:2000, Fujifilm Wako, Osaka, Japan, 019-19741), CD3 (1:1000; Agilent Technologies, CA, USA, IR503), KDM2B (1:50; Santa Cruz Biotechnology, Inc.,sc-293279), CD31 (1:250; Abcam, JC/70A), PD-L1 (1:100; clone 6C11-3A11), and CD204 (1:800; Medicinal Chemistry Pharmaceutical Co., Ltd., Sapporo, Japan, KT022) as described previously ${ }^{7}$. Nano Zoomer 2.0-RS (Hamamatsu Photonics, Hamamatsu, Japan) was used to scan histological slides, which were then processed in QuPath ver 0.2.1 ${ }^{34}$. Scanned slides were opened in QuPath as Brightfield (H-DAB), and the Estimate Stain Vectors feature was used to automatically adjust the staining colors. Normal endothelial and tumor cells were detected using the Cell Detection function. The Create Detection Classifiers function was used to label cells based on their morphologies and locations, allowing 
the QuPath software to correctly classify each cell type. The collected data was exported and used for further analysis.

Immunofluorescence assay. Tissue samples were fixed, processed, and incubated with primary antibodies for Ibal and PD-L1 as described above. Tissue samples were washed with PBS three times for 5 min each before adding a secondary goat anti-rabbit IgG H \& L (1:1000, Alexa Fluor 555, Abcam, ab150078) and Goat Anti-Rat IgG H \& L (1:1000, Alexa Fluor 488, Abcam, ab150157) in 5\% skim milk for $1 \mathrm{~h}$ at RT. Sections were washed with PBS three times for 5 min each, mounted with DAPI-containing mounting medium, and covered with $24 \times 32 \mathrm{~mm}$ coverslip. Tissues were examined under a confocal microscope (LSM700, Carl Zwiss, Oberkochen, German).

Statistical analysis. Statistical analyses were performed with Microsoft Excel and R software (version 3.6.3). Student's $t$-test and Mann-Whitney $U$ test were used to analyze the difference between two groups, while Tukey's test was used to analyze differences between multiple groups. $P$-values less than 0.05 were considered statistically significant.

\section{Data availability}

The datasets generated during and/or analyzed during the current study are available from the corresponding author on reasonable request.

Received: 2 August 2021; Accepted: 25 January 2022

Published online: 08 February 2022

\section{References}

1. Spangler, W. L. \& Culbertson, M. R. Prevalence, type, and importance of splenic diseases in dogs: 1,480 cases (1985-1989). J. Am. Vet. Med. Assoc. 200, 829-834 (1992).

2. Prymak, C., McKee, L. J., Goldschmidt, M. H. \& Glickman, L. T. Epidemiologic, clinical, pathologic, and prognostic characteristics of splenic hemangiosarcoma and splenic hematoma in dogs: 217 cases (1985). J. Am. Vet. Med. Assoc. 193, 706-712 (1988).

3. Johannes, C. M. et al. Hemangiosarcoma in cats: 53 cases (1992-2002). J. Am. Vet. Med. Assoc. 231, 1851-1856 (2007).

4. Southwood, L. L. et al. Disseminated hemangiosarcoma in the horse: 35 cases. J. Vet. Intern. Med. 14, 105-109 (2000).

5. Cohen, S. M. et al. Hemangiosarcoma in rodents: Mode-of-action evaluation and human relevance. Toxicol. Sci. 111, 4-18 (2009).

6. Young, R. J., Brown, N. J., Reed, M. W., Hughes, D. \& Woll, P. J. Angiosarcoma. Lancet Oncol. 11, 983-991 (2010).

7. Gulay, K. C. M. et al. KDM2B promotes cell viability by enhancing DNA damage response in canine hemangiosarcoma. J. Genet. Genomics 48, 618-630 (2021).

8. Lei, Z. G., Ren, X. H., Wang, S. S., Liang, X. H. \& Tang, Y. L. Immunocompromised and immunocompetent mouse models for head and neck squamous cell carcinoma. Onco Targets Ther. 9, 545 (2016).

9. Masuzawa, M. et al. Establishment of a new murine-phenotypic angiosarcoma cell line (ISOS-1). J. Dermatol. Sci. 16, 91-98 (1998).

10. Toda, K. et al. Establishment and characterization of a tumorigenic murine vascular endothelial cell line (F-2). Cancer Res. 50, 5526-5530 (1990).

11. Allavena, P., Sica, A., Garlanda, C. \& Mantovani, A. The Yin-Yang of tumor-associated macrophages in neoplastic progression and immune surveillance. Immunol. Rev. 222, 155-161 (2008).

12. Chen, Y. et al. Tumor-associated macrophages: An accomplice in solid tumor progression. J. Biomed. Sci. 26, 1-13 (2019).

13. Wang, Y. \& Harris, D. C. H. Macrophages in renal disease. J. Am. Soc. Nephrol. 22, 21-27 (2011).

14. Nagano, M. et al. CD204-positive macrophages accumulate in breast cancer tumors with high levels of infiltrating lymphocytes and programmed death ligand-1 expression. Oncol. Lett. 21, 36 (2021).

15. Kubota, K. et al. $\mathrm{CD} 163^{+} \mathrm{CD} 204^{+}$tumor-associated macrophages contribute to $\mathrm{T}$ cell regulation via interleukin-10 and $\mathrm{PD}-\mathrm{L} 1$ production in oral squamous cell carcinoma. Sci. Rep. 7, 1755 (2017).

16. Fitzgerald, M. L., Moore, K. J., Freeman, M. W. \& Reed, G. L. Lipopolysaccharide induces scavenger receptor A expression in mouse macrophages: A divergent response relative to human THP-1 monocyte/macrophages. J. Immunol. 164, 2692-2700 (2000).

17. Hashimoto, R. et al. LPS enhances expression of CD204 through the MAPK/ERK pathway in murine bone marrow macrophages. Atherosclerosis 266, 167-175 (2017).

18. Kitajima, T., Iwashiro, M., Kuribayashi, K. \& Imamura, S. Immunological characterization of tumor-rejection antigens on ultraviolet-light-induced tumors originating in the CB6F1 mouse. Cancer Immunol. Immunother. 6, 372-378 (1994).

19. Zhou, L., Jilderda, L. J. \& Foijer, F. Exploiting aneuploidy-imposed stresses and coping mechanisms to battle cancer. Open Biol. 10, 200148 (2020).

20. Maekawa, N. et al. Immunohistochemical analysis of PD-L1 expression in canine malignant cancers and PD-1 expression on lymphocytes in canine oral melanoma. PLoS One 11, e0157176 (2016).

21. Maekawa, N. et al. A canine chimeric monoclonal antibody targeting PD-L1 and its clinical efficacy in canine oral malignant melanoma or undifferentiated sarcoma. Sci. Rep. 7, 8951 (2017).

22. Maekawa, N. et al. PD-L1 immunohistochemistry for canine cancers and clinical benefit of anti-PD-L1 antibody in dogs with pulmonary metastatic oral malignant melanoma. npj Precis. Oncol. 5, 1-9 (2021).

23. Kitamura, T. et al. Proinsulin C-peptide activates cAMP response element-binding proteins through the p38 mitogen-activated protein kinase pathway in mouse lung capillary endothelial cells. Biochem. J. 366, 737-744 (2002).

24. Curtis, A. S. G. \& Renshaw, R. M. Lymphocyte-endothelial interactions and histocompatibility restriction. In In Vivo Immunology Histophysiology of the Lymphoid System (ed. Nieuwenhuis, P.) 193-198 (Springer US, 2012).

25. Raschke, W. C., Baird, S., Ralph, P. \& Nakoinz, I. Functional macrophage cell lines transformed by Abelson leukemia virus. Cell 15, 261-267 (1978).

26. Murai, A. et al. Constitutive phosphorylation of the mTORC2/Akt/4E-BP1 pathway in newly derived canine hemangiosarcoma cell lines. BMC Vet. Res. 8, 128 (2012).

27. Harasawa, R. et al. Detection and tentative identification of dominant mycoplasma species in cell cultures by restriction analysis of the 16S-23S rRNA intergenic spacer regions. Res. Microbiol. 144, 489-493 (1993).

28. Harasawa, R. et al. Rapid detection and differentiation of the major mycoplasma contaminants in cell cultures using real-time PCR with SYBR green I and melting curve analysis. Microbiol. Immunol. 49, 859-863 (2005).

29. Wang, J., Niu, N., Xu, S. \& Jin, Z. G. A simple protocol for isolating mouse lung endothelial cells. Sci. Rep. 9, 1458 (2019).

30. DeCicco-Skinner, K. L. et al. Endothelial cell tube formation assay for the in vitro study of angiogenesis. J. Vis. Exp. 91, e51312 (2014). 
31. Schneider, C. A., Rasband, W. S. \& Eliceiri, K. W. NIH Image to ImageJ: 25 years of image analysis. Nat. Methods 9, 671-675 (2012).

32. Vidyarthi, A. et al. TLR-3 stimulation skews M2 macrophages to M1 through IFN- $\alpha \beta$ signaling and restricts tumor progression. Front. Immunol. 9, 1650 (2018)

33. Chen, Y. et al. SAK-HV promotes RAW264.7 cells migration mediated by MCP-1 via JNK and NF- $\kappa$ B pathways. Int. J. Biol. Sci. 14, 1993-2002 (2018).

34. Bankhead, P. et al. QuPath: Open source software for digital pathology image analysis. Sci. Rep. 7, 16878 (2017).

\section{Acknowledgements}

We would like to extend our sincerest gratitude to Dr. Kazuhiro Kimura (Hokkaido University) and Dr. Hiroki Sakai (Gifu University) for providing LEII cell line and canine hemangiosarcoma cell lines, respectively. We are grateful to the members of the Laboratory of Comparative Pathology, Faculty of Veterinary Medicine, Hokkaido University for their invaluable support during the conduct of this study. This research was supported by the KAKENHI Grant-in-Aid for Young Scientist (KA, 20K15654) provided by Japan Society for the Promotion of Science, and by a research grant provided by the Kuribayashi Foundation (KA, No. 2-2).

\section{Author contributions}

K.G. Conceptualization, Methodology, Investigation, Formal Analysis, Data Curation, Writing-Original Draft, Writing-Review and Editing. K.A. Conceptualization, Methodology, Investigation, Formal Analysis, Data Curation, Writing-Review and Editing, Supervision, Funding acquisition. N.M. Investigation, methodology, supervision. T.S. Investigation. S.K. Methodology, Supervision. A.K. Writing-Review and Editing. T.K. Supervision.

\section{Competing interests}

The authors declare no competing interests.

\section{Additional information}

Supplementary Information The online version contains supplementary material available at https://doi.org/ 10.1038/s41598-022-06203-w.

Correspondence and requests for materials should be addressed to K.A.

Reprints and permissions information is available at www.nature.com/reprints.

Publisher's note Springer Nature remains neutral with regard to jurisdictional claims in published maps and institutional affiliations.

(1) Open Access This article is licensed under a Creative Commons Attribution 4.0 International License, which permits use, sharing, adaptation, distribution and reproduction in any medium or format, as long as you give appropriate credit to the original author(s) and the source, provide a link to the Creative Commons licence, and indicate if changes were made. The images or other third party material in this article are included in the article's Creative Commons licence, unless indicated otherwise in a credit line to the material. If material is not included in the article's Creative Commons licence and your intended use is not permitted by statutory regulation or exceeds the permitted use, you will need to obtain permission directly from the copyright holder. To view a copy of this licence, visit http://creativecommons.org/licenses/by/4.0/.

(C) The Author(s) 2022 